\title{
Insectos y ácaros perjudiciales en los arboricultivos de la Universidad Nacional Agraria La Molina, Perú
}

\author{
Harmful insects and Acarina in the urban species trees of La Molina National \\ Agraria University, Peru
}

\author{
Maricel J. Móstiga Rodríguez', Pedro W. Lozada Robles ${ }^{2}$
}

\begin{abstract}
RESUMEN:
Durante un periodo de un año en el campus de la Universidad Nacional Agraria La Molina se evaluó la incidencia y severidad del ataque de 16 insectos picadores-chupadores, masticadores y un ácaro en 24 especies forestales cultivadas en la ciudad de Lima, usando el método de Agrios (2002) modificada por Manta (2013), siendo Aleurodicus juleikae la especie con mayor número de hospederos. Estos agentes han sido clasificados en 3 grupos: 7 plagas clave, 9 plagas potenciales y 4 plagas esporádicas. Quince de estos agentes fueron reportados por primera vez para el hospedero forestal en el Museo de Historia Natural de la Universidad Nacional Mayor de San Marcos y en el Servicio Nacional de Sanidad Agraria- Perú, siendo Glycaspis brimblecombei en Eucalyptus spp. el primer registro para Lima y Neophyllaphis araucariae, Platycorypha nigrivirga y Singhiella simplex en Araucaria excelsa, Tipuana tipu y Ficus benjamina respectivamente los primeros registros para Perú.
\end{abstract}

Palabras clave: Incidencia, severidad, picadores-chupadores, plagas, cultivos forestales.

\begin{abstract}
:
Over a period of 1 years on the campus of the La Molina National Agrarian University, the incidence and severity of the attack of 16 sucking and chewing insects and one Acarina were identified and evaluated in 24 forest species grown in urban environments representative of the city of Lima. These agents are grouped into 3 types of pests: 7 keys, 9 potentials and 4 sporadic. Fifteen of these insects were reported for the first time for the plant host in the Natural History Museum of the San Marcos National University and in the National Agrarian Health Service (SENASA Perú), being Glycaspis brimblecombei in Eucalyptus spp. first record for Lima and Neophyllaphis araucariae, Platycorypha nigrivirga $y$ Singhiella simplex in Araucaria excelsa, Tipuana tipu $y$ Ficus benjamina respectively, first records from Peru.
\end{abstract}

Key words: Incidence, severity, sucking, pests, forest species.

\section{Introducción}

Principalmente son tres estudios los que enmarcan a la entomología forestal Peruana. El primero de ellos se realizó por Dourojeanni (1963) quien señala los principales grupos de insectos que causan perjuicio a la actividad forestal y se muestran algunas observaciones sobre su biología, daño, huéspedes y control natural.

El segundo, por Alata (1973), en una recopilación de la información referente a plagas insectiles reúne a
25 especies forestales y 18 ornamentales y genera una lista donde menciona a los insectos cuyos hospederos han sido especies forestales (95), corteza (13), madera (5) y troncos (26).

El tercero, por Servicio Nacional de Sanidad Agraria (SENASA) (2008) junto a la Universidad Nacional Agraria La Molina (UNALM) (Lima, Perú) y otras universidades generan información sobre 11 insectos forestales provenientes de bosques naturales.

Al ser escasos los estudios que presentan el daño que se producen en especies forestales en términos

1 Facultad de Ciencias Forestales, Universidad Nacional Agraria La Molina, Lima, PERÚ.

2 Departamento de Entomología, Museo de Historia Natural, UNMSM, Apartado 14-0434, Lima, PERÚ.

* Autor por correspondencia: mmostiga@lamolina.edu.pe; plozada57@hotmail.com

Fecha de Recepción: 15 noviembre, 2018.

Fecha de Aceptación: 5 febrero, 2019.

DOI: http://dx.doi.org/10.4067/S0718-34292019005000503. Publicado en línea: 14-junio-2019. 
cuantitativos así como la determinación taxonómica de los insectos y ácaros causantes de daño en los arboricultivos limeños, el objetivo de la investigación es evaluar cualitativamente y cuantitativamente los síntomas y signos presentes en las principales especies de árboles del campus de la UNALM.

\section{Materiales y Métodos}

\section{Área de estudio}

El estudio se realizó en el campus de la UNALM, ubicado en la ciudad de Lima, distrito de La Molina. Geográficamente se encuentra entre las coordenadas $12^{\circ} 05^{\prime \prime}$ Latitud Sur y $76^{\circ} 56^{\prime \prime}$ Longitud Oeste a $251 \mathrm{msnm}$. Según la clasificación de Zonas de Vida de Holdridge el área de estudio se ubica en la formación ecológica Desierto desecado subtropical (dd - S).

Los datos meteorológicos de las evaluaciones fueron obtenidos del Observatorio Meteorológico Alexander Von Humboldt y se presentan en la Tabla 1.

Tabla 1. Datos Meteológicos del Periodo de Evaluación

\begin{tabular}{lllll}
\hline & $\begin{array}{c}\text { Agosto } \\
\mathbf{2 0 1 1}\end{array}$ & $\begin{array}{c}\text { Noviembre } \\
\mathbf{2 0 1 1}\end{array}$ & $\begin{array}{c}\text { Febrero } \\
\mathbf{2 0 1 2}\end{array}$ & $\begin{array}{c}\text { Mayo } \\
\mathbf{2 0 1 2}\end{array}$ \\
\hline $\begin{array}{l}\text { Temperatura } \\
\text { Máxima }\left(\mathrm{C}^{\circ}\right)\end{array}$ & 17,5 & 23 & 29,2 & 29,7 \\
\hline $\begin{array}{l}\text { Temperatura } \\
\text { Mínima }\left(\mathrm{C}^{\circ}\right)\end{array}$ & 13,5 & 15,3 & 19,7 & 15,8 \\
\hline $\begin{array}{l}\text { Temperatura } \\
\text { Promedio }\left(\mathrm{C}^{\circ}\right)\end{array}$ & 15,4 & 19,4 & 25,3 & 20,2 \\
\hline $\begin{array}{l}\text { Humedad } \\
\text { Relativa } \\
\text { Máxima (\%) }\end{array}$ & 97 & 93 & 93 & 94 \\
\hline $\begin{array}{l}\text { Humedad } \\
\text { Relativa } \\
\text { Mínima (\%) }\end{array}$ & 83 & 72 & & \\
\hline $\begin{array}{l}\text { Humedad } \\
\text { Relativa } \\
\text { Promedio (\%) }\end{array}$ & 90 & 82 & 60 & 73 \\
\hline
\end{tabular}

\section{Población y muestra}

La población es el arbolado del campus de la UNALM, del que se tomó una muestra de 406 árboles (23\% de la población). La muestra agrupa 24 especies forestales mayores de $10 \mathrm{~cm}$. de diámetro a la altura del pecho- DAP (Tabla 2).

\section{Evaluación}

Debido a que el agente causal de daño en el hospedero varía a lo largo del año calendario,
Tabla 2. Lista de especies y árboles seleccionados para la evaluación

\begin{tabular}{|c|c|c|c|}
\hline Especie forestal & $\begin{array}{l}\quad \mathrm{N}^{\mathbf{o}} \\
\text { Total de } \\
\text { árboles }\end{array}$ & $\begin{array}{c}\mathbf{N}^{\circ} \\
\text { árboles } \\
\text { evaluados }\end{array}$ & $\begin{array}{c}\% \text { árboles } \\
\text { evaluados/ } \\
\text { total de la } \\
\text { especie }\end{array}$ \\
\hline $\begin{array}{l}\text { Grevillea robusta A. Cunn. ex } \\
\text { R. Br. }\end{array}$ & 198 & 30 & 15 \\
\hline Schinus molle L. & 177 & 34 & 19 \\
\hline Casuarina cunninghamiana Miq. & 144 & 23 & 16 \\
\hline Eucalyptus spp. & 132 & 28 & 21 \\
\hline Ficus benjamina $\mathrm{L}$. & 126 & 24 & 19 \\
\hline Salix humboldtiana Willd. & 111 & 24 & 22 \\
\hline Tecoma spp. & 104 & 23 & 22 \\
\hline Tipuana tipu (Benth.) Kuntze & 98 & 24 & 24 \\
\hline Spathodea campanulata P. Beauv. & 94 & 23 & 24 \\
\hline Acacia retinoides & 69 & 18 & 26 \\
\hline Delonix regia (Bojer ex Hook.) Raf. & 61 & 20 & 33 \\
\hline Schinus terebinthifolius Raddi & 54 & 16 & 30 \\
\hline Cedrela odorata $\mathrm{L}$. & 54 & 12 & 22 \\
\hline Koelreuteria paniculata Laxm. & 54 & 12 & 22 \\
\hline Jacaranda mimosifolia D. Don & 48 & 16 & 33 \\
\hline $\begin{array}{l}\text { Enterolobium cyclocarpum } \\
\text { (Jacq.) Griseb. }\end{array}$ & 38 & 7 & 18 \\
\hline $\begin{array}{l}\text { Triplaris peruviana Fisch. \& E. } \\
\text { Mey. ex C.A. Mey. }\end{array}$ & 37 & 5 & 14 \\
\hline Fraxinus americana $\mathrm{L}$. & 36 & 6 & 17 \\
\hline Parkinsonia aculeata L. & 27 & 14 & 52 \\
\hline Melia azedarach $\mathrm{L}$. & 22 & 13 & 59 \\
\hline Lagerstroemia speciosa (L.) Pers. & 18 & 10 & 56 \\
\hline Araucaria excelsa (Lamb.) R. Br. & 13 & 10 & 77 \\
\hline $\begin{array}{l}\text { Cupressus macrocarpa Hartw. } \\
\text { ex Gordon }\end{array}$ & 11 & 8 & 73 \\
\hline Swietenia macrophylla King & 6 & 6 & 100 \\
\hline Total & 1732 & 406 & 23,44 \\
\hline
\end{tabular}

se realizó una evaluación por cada estación: invierno 2011, primavera 2011, verano 2012 y otoño 2012.

Previo a cada evaluación en los árboles seleccionados se distinguieron a los insectos y ácaros fitófagos presentes, y se diseñaron escalas y formatos de evaluación. Estas evaluaciones se dividieron en tres partes: evaluación cualitativa, evaluación de las condiciones que predisponen el ataque de insectos y ácaros y evaluación cuantitativa.

\section{Evaluación cualitativa}

La evaluación cualitativa consistió en la observación, colección y descripción de síntomas y signos, además del análisis de la biología y ecología 
de los insectos y ácaros, las cuales se realizaron siguiendo la metodología de SENASA (2006) y Manta (2013), diagnosticando al agente causal.

La identificación de las muestras de insectos o ácaros se realizaron en el Museo de Historia Natural (MUSM) de la Universidad Nacional Mayor de San Marcos, usando literatura taxonómica especializada, la comparación con los especímenes existentes en la colección, según la descripción morfológica de artículos entomológicos o enviando fotos e individuos a especialistas en el extranjero.

\section{Evaluación cuantitativa}

La evaluación cuantitativa consistió en el cálculo de la incidencia y severidad del síntoma, de acuerdo a la metodología definida por Agrios (2002) adaptada por Manta (2013) a través de las siguientes fórmulas:

$$
\mathrm{I}=\frac{\mathrm{n1^{+ }} n 2^{+} n 3^{+} n 4^{+} n 5^{+} n 6^{+} \ldots{ }^{+} n n}{\mathrm{~N}}
$$

Donde:

I: Incidencia del sintoma o signo

n1,n2,n3,n4,n5,n6,...,nn: número de árboles con el síntoma o signo

$\mathrm{N}$ : número total de árboles observados

Manta (2013), señala que la incidencia, es el número o proporción de árboles con el síntoma o signo respecto al total evaluado y es expresado en porcentaje.

\section{S: $\frac{1 n 1^{+} 2 n 2^{+} 3 n 3^{+} 4 n 4^{+} \ldots+{ }^{+} X n x}{N}$}

S: grado de daño

Grado: $1,2,3,4, \ldots, X$ (escala a fijar por el investigador según el agente causal)

n1,n2,n3,n4,..,nx: número de árboles o de partes del árbol con el síntoma o signo.

$\mathrm{N}$ : número total de árboles o número total de partes observadas.

Dentro de la evaluación cuantitativa se considera la clasificación de los síntomas causados por los insectos o ácaros encontrados en la muestra, de acuerdo a los valores de incidencia y severidad y su duración (Manta 2013). Para la clasificación de plagas se usó la definición de evaluación cuantitativa de Manta (2013) y una adaptación de la clasificación de Delgado y Couturier (2004), con la cual se agrupó a los insectos y ácaros en tres grupos de: plagas claves, plagas potenciales y plagas esporádicas u ocasionales.

Se consideran plagas claves a aquellos insectos y ácaros cuya distribución espacial representada por la incidencia es mayor o igual al $50 \%$ y su grado de severidad mayor a 2 durante cualquiera de las estaciones del año, y su ataque se extienda de tres a cuatro estaciones en el año.

Como plagas esporádicas u ocasionales a aquellos insectos o ácaros cuyo ataque es de una a dos estaciones e incidencia analizada espacialmente es mayor igual al 50\%.

Y finalmente, como plagas potenciales a aquellos insectos o ácaros cuya distribución espacial representada por la incidencia es mayor o igual al $50 \%$ o su grado de severidad mayor o igual a 2 durante cualquiera de las estaciones del año. Estos insectos y ácaros se encuentran solo en algunas zonas del campus posiblemente a causa de algún factor predisponente en dichas zonas del campus.

\section{Resultados}

Se identificaron un ácaro fitófago y 16 insectos fitófagos, atacando el follaje de las especies forestales (Tabla 3).

La Tabla 4 muestra a los insectos y ácaros clasificados de acuerdo a la incidencia, severidad, duración del ataque y por tipo de plaga. De acuerdo a los niveles de incidencia, severidad y duración se clasificó en 7 plagas claves (6 insectos y 1 ácaro), 4 plagas esporádicas u ocasionales (4 insectos) y 9 plagas potenciales (9 insectos). Es importante mencionar que las plagas clave no logran evidenciar su ataque a simple vista en la copa del árbol a excepción de Erosina hyberniata, Gynaikothrips uzeli y Singhiella simplex.

Respecto al hábito de alimentación de los insectos y ácaros se reconoce 8 monófagas (Figura 2) y 9 polífagas (Figura 1) (Tabla 5). Las especies polífagas tienden a atacar a hojas maduras más que a las tiernas. Mientras que las especies monófagas tienden a atacar hojas jóvenes, salvo en el caso de especies que pueden atacar a otras especies vegetales, tal como lo indica Pérez (1999).

En la Tabla 6 se presenta al hospedero y el tipo de pieza bucal que le causa daño al árbol cuando se alimenta de él. Así Araucaria excelsa, Swietenia macrophylla, Cedrela odorata, Eucalyptus spp., Ficus benjamina, Grevillea robusta, Tecoma spp., Lagerstromia speciosa, Schinus terebinthifolius, Acacia retinoides, Schinus molle, Salix humboldtiana, Tipuana tipu y Spathodea campanulata presentaron daños como comeduras en 
Tabla 3. Clasificación taxonómica, hospederos e identificación de los insectos y ácaros

\begin{tabular}{|c|c|c|c|c|c|}
\hline Clase & Orden & Familia & Especie & Nombre común & Hospederos \\
\hline \multirow{16}{*}{ Insecta } & \multirow{3}{*}{ Lepidoptera } & Geometridae & Erosina hyberniata Guenée, 1957. (Figura 2A) & Larva del tecoma & Tecoma spp. \\
\hline & & Lycaenidae & Pseudolycaena nellyae Lamas, 1975. (Figura 1A) & Gusano verde del brote & Acacia retinoides \\
\hline & & Pyralidae & Marasmia trapezalis Guenée, 1854. (Figura 1E) & Enrollador de hojas & Acacia retinoides \\
\hline & \multirow{12}{*}{ Hemiptera } & \multirow{2}{*}{ Diaspididae } & Aspidiotus nerii, Bouché 1933. (Figura 1B) & Escama blanca & $\begin{array}{l}\text { Grevillea robusta } \\
\text { Acacia retinoides } \\
\text { Salix humboldtiana }\end{array}$ \\
\hline & & & Pinnaspis aspidistrae (Signoret, 1869) (Figura 1G) & Piojo blanco & $\begin{array}{l}\text { Tipuana tipu y Schinus } \\
\text { terebinthifolius. }\end{array}$ \\
\hline & & & Parasaissetia nigra (Nietner, 1861) (Figura 1H) & Cochinilla negra & $\begin{array}{l}\text { Ficus bejamina } \\
\text { Schinus terebinthifolius }\end{array}$ \\
\hline & & onerate & Ceroplastes cirripediformis Comstock, 1881. (Figura 1I) & Cochinilla blanca & $\begin{array}{l}\text { Ficus benjamina } \\
\text { Schinus terebinthifolius. }\end{array}$ \\
\hline & & Tingidae & Leptobyrsa decora Drake, 1922. (Figura 1C) & Chinche de encaje & Tecoma spp. \\
\hline & & \multirow[t]{3}{*}{ Aleyrodidae } & Aleurodicus juleikae Bondar, 1933. (Figura 1D) & Mosca blanca gigante & $\begin{array}{l}\text { Ficus benjamina } \\
\text { Eucalyptus spp. } \\
\text { Lagerstromia speciosa Grevillea robusta } \\
\text { Tecoma spp. } \\
\text { Schinus terebinthifolius } \\
\text { Schinus molle } \\
\text { Tipuana tipu }\end{array}$ \\
\hline & & & $\begin{array}{l}\text { Aleurodicus coccolobae Quaintance \& Baker, } 1914 . \\
\text { (Figura 1F) }\end{array}$ & Mosca blanca del cocotero & $\begin{array}{l}\text { Acacia retinoides } \\
\text { Salix humboldtiana }\end{array}$ \\
\hline & & & Singhiella simplex (Singh, 1931) (Figura 2E) & Mosca blanca del ficus & Ficus benjamina \\
\hline & & Calophyidae & Calophya schini Tuthill, 1959. (Figura 2C) & Psílido del molle & Schinus molle \\
\hline & & \multirow{2}{*}{ Psyllidae } & Glycaspis brimblecombei Moore, 1964. (Figura 2G) & Psílido del eucalipto rojo & Eucalyptus spp. \\
\hline & & & Platycorypha nigrivirga Burckhardt, 1987. (Figura 2D) & Psílido de la tipa & Tipuana Tipu \\
\hline & & Aphididae & Neophyllaphis araucariae Takahashi, 1937. (Figura 2B) & Pulgón de la araucaria & Araucaria excelsa \\
\hline & Thysanoptera & Phloeothripidae & Gynaikothrips uzeli Zimerman, 1909. (Figura 2F) & Thrips & Ficus benjamina \\
\hline Arachnida & Trombidiformes & Tetranychidae & Schizotetranychus sp. (Figura 2H) & Ácaro del sauce & Salix humboldtiana. \\
\hline
\end{tabular}

Tabla 4. Incidencia, severidad y duración de las clases de insectos y ácaros plaga

\begin{tabular}{|c|c|c|c|c|c|c|c|c|c|c|c|}
\hline \multirow[b]{2}{*}{ Hospedero } & \multirow[b]{2}{*}{ Insectos y ácaros } & \multicolumn{4}{|c|}{ INCIDENCIA } & \multicolumn{4}{|c|}{ SEVERIDAD } & \multirow{2}{*}{ DURACIÓN } & \multirow{2}{*}{ Tipo de plaga } \\
\hline & & $I$ & $\boldsymbol{P}$ & $\boldsymbol{V}$ & $O$ & $I$ & $P$ & $\boldsymbol{V}$ & $O$ & & \\
\hline Tecoma spp. & Erosina hyberniata & $96 \%$ & $60 \%$ & $60 \%$ & $30 \%$ & 4 & 3 & 3 & 2 & 4 estaciones & \multirow{7}{*}{ Plaga clave } \\
\hline Ficus benjamina & Aleurodicus juleikae & $71 \%$ & $75 \%$ & $17 \%$ & $50 \%$ & 3 & 4 & 2 & 3 & 4 estaciones & \\
\hline Schinus molle & Calophya schini & $91 \%$ & $82 \%$ & $35 \%$ & $29 \%$ & 3 & 2 & 2 & 2 & 4 estaciones & \\
\hline Eucalyptus sp. & Aleurodicus juleikae & $54 \%$ & $43 \%$ & $25 \%$ & $39 \%$ & 3 & 2 & 1 & 2 & 4 estaciones & \\
\hline Lagerstromia speciosa & Aleurodicus juleikae & $0 \%$ & $40 \%$ & $50 \%$ & $90 \%$ & 1 & 1 & 2 & 3 & 3 estaciones & \\
\hline Acacia retinoides & Pseudolycaena nellyae & $78 \%$ & $67 \%$ & $22 \%$ & $0 \%$ & 2 & 3 & 1 & 1 & 3 estaciones & \\
\hline Salix humboldtiana & Schizotetranychus sp. & $71 \%$ & $54 \%$ & $4 \%$ & $0 \%$ & 3 & 3 & 1 & 1 & 3 estaciones & \\
\hline Araucaria excelsa & $\begin{array}{l}\text { Neophyllaphis } \\
\text { araucariae }\end{array}$ & $0 \%$ & $50 \%$ & $80 \%$ & $0 \%$ & \multicolumn{4}{|c|}{ Sin evaluación de severidad } & 2 estaciones & \multirow{4}{*}{$\begin{array}{l}\text { Plaga } \\
\text { esporádica } \\
\text { u ocasional }\end{array}$} \\
\hline Tecoma sp & Leptobyrsa decora & $0 \%$ & $17 \%$ & $17 \%$ & $0 \%$ & 1 & 1 & 1 & 1 & 2 estaciones & \\
\hline Tipuana tipu & Platycorypha nigrivirga & $0 \%$ & $0 \%$ & $38 \%$ & $0 \%$ & \multicolumn{4}{|c|}{ Sin evaluación de severidad } & 1 estación & \\
\hline Eucalyptus sp. & $\begin{array}{l}\text { Glycaspis } \\
\text { brimblecombei }\end{array}$ & $0 \%$ & $0 \%$ & $11 \%$ & $0 \%$ & 1 & 1 & 1 & 1 & 1 estación & \\
\hline Schinus terebinthifolius & Aleurodicus juleikae & $81 \%$ & $81 \%$ & $25 \%$ & $25 \%$ & 2 & 2 & 1 & 2 & 4 estaciones & \multirow{9}{*}{$\begin{array}{l}\text { Plaga } \\
\text { potencial }\end{array}$} \\
\hline Grevillea robusta & Aspidiotus nerii & $70 \%$ & $23 \%$ & $23 \%$ & $17 \%$ & 2 & 1 & 1 & 1 & 4 estaciones & \\
\hline Acacia retinoides & Marasmia trapezalis & $17 \%$ & $44 \%$ & $44 \%$ & $0 \%$ & 1 & 3 & 3 & 1 & 3 estaciones & \\
\hline Spathodea campanulata & Agalla en las hojas & $17 \%$ & $65 \%$ & $9 \%$ & $22 \%$ & 1 & 2 & 1 & 2 & 4 estaciones & \\
\hline Ficus benjamina & Gynaikothrips uzeli & $54 \%$ & $42 \%$ & $0 \%$ & $29 \%$ & 2 & 2 & 1 & 2 & 3 estaciones & \\
\hline Cedrela odorata & Comedura en hojas & $17 \%$ & $8 \%$ & $50 \%$ & $50 \%$ & 1 & 1 & 2 & 2 & 4 estaciones & \\
\hline Salix humboldtiana & Comedura en hojas & $54 \%$ & $33 \%$ & $4 \%$ & $0 \%$ & 2 & 1 & 1 & 1 & 3 estaciones & \\
\hline Schinus terebinthifolius & Pinnaspis aspidistrae & $63 \%$ & $56 \%$ & $0 \%$ & $0 \%$ & 2 & 1 & 1 & 1 & 2 estaciones & \\
\hline Acacia retinoides & Aleurodicus juleikae & $22 \%$ & $6 \%$ & $6 \%$ & $50 \%$ & 1 & 1 & 1 & 1 & 4 estaciones & \\
\hline
\end{tabular}

Donde: I: invierno, P: primavera, V: verano y O: otoño; Grado= 1: la escala mínima y 5: la escala máxima 

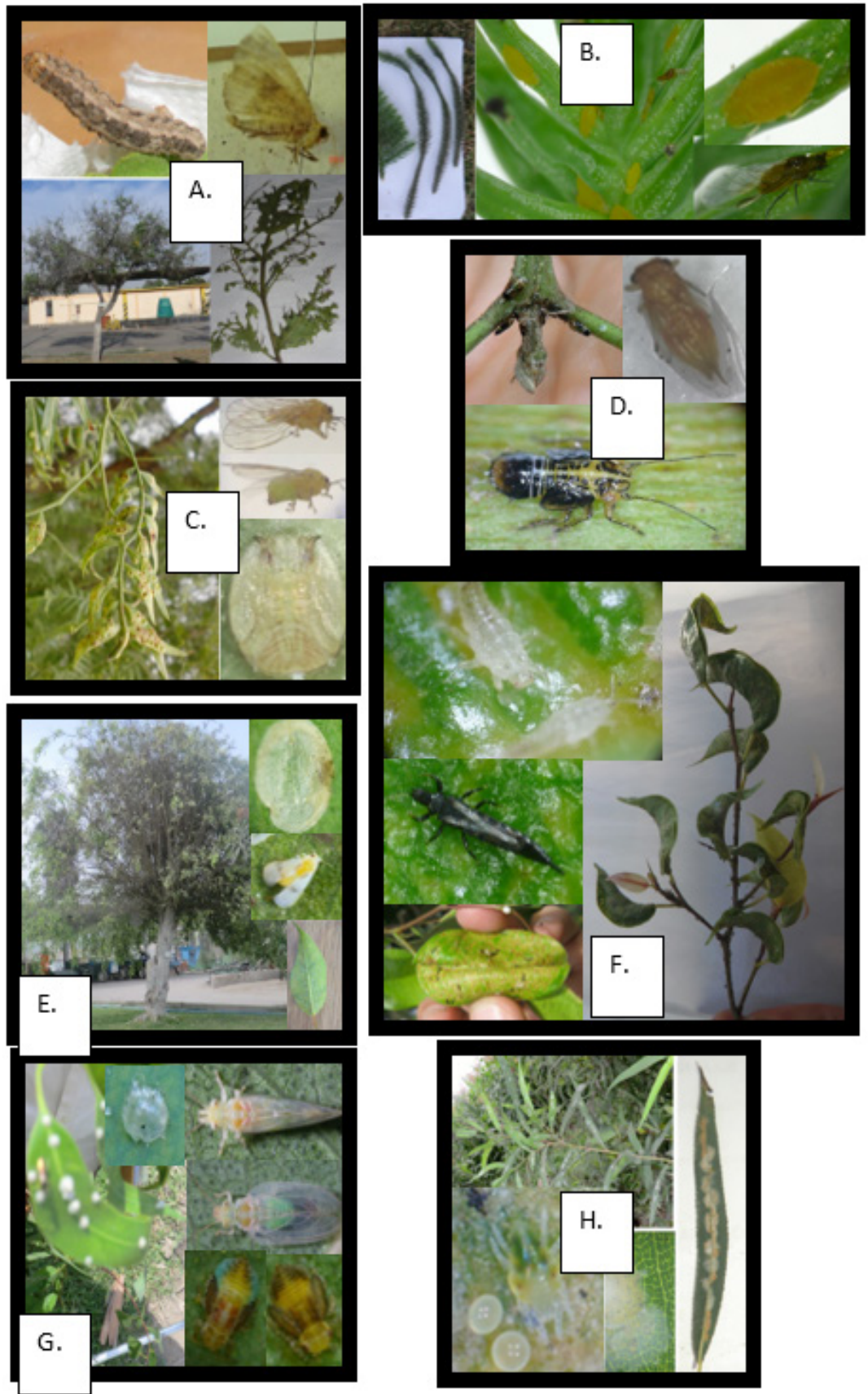

Figura 2. Síntomas y signos de los insectos y ácaro monofagos. A. Erosina hyberniata; B. Neophyllaphis araucariae; C. Calophya schini; D. Platycorypha nigrivirga; E. Singhiella simplex; F. Gynaikothrips uzeli; G. Glycaspis brimblecombei y H. Schizotetranychus sp. 


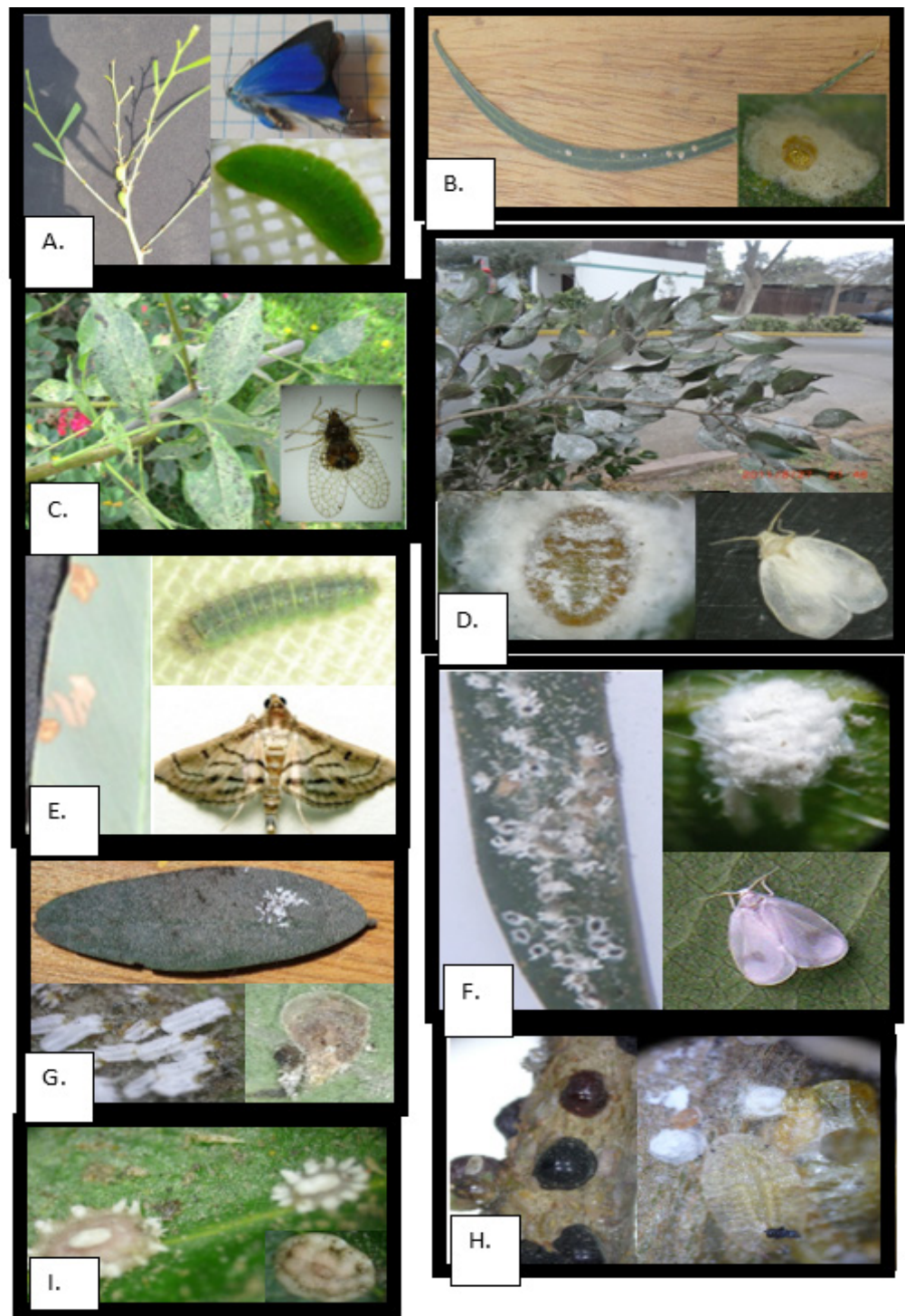

Figura 1. Síntomas y signos de los insectos polífagos.A. Pseudolycaena nellyae; B. Aspidiotus nerii; C. Leptobyrsa decora; D. Aleurodicus juleikae; E. Marasmia trapezalis; F. Aleurodicus coccolobae; G. Pinnaspis aspidistrae; H. Parasaissetia nigra; y I. Ceroplastes cirripediformis

Tabla 5. Clasificación de los insectos y ácaros por tipo de piezas bucales y hábito de alimentación

\begin{tabular}{|c|c|c|}
\hline \multirow{2}{*}{ Tipo de piezas bucales } & \multicolumn{2}{|l|}{ Insectos y ácaros } \\
\hline & Monófago & Polífago \\
\hline \multirow{2}{*}{ Masticador } & \multirow[t]{2}{*}{ Erosina hyberniata } & Pseudolycaena nellyae \\
\hline & & Marasmia trapezalis \\
\hline \multirow{7}{*}{ Picador- Chupador } & Glycaspis brimblecombei & Aspidiotus nerii \\
\hline & Platycorypha nigrivirga & Pinnaspis aspidistrae \\
\hline & Neophyllaphis araucariae & Parasaissetia nigra \\
\hline & Gynaikothrips uzeli & Ceroplastes cirripediformis \\
\hline & Singhiella simplex & Leptobyrsa decora \\
\hline & Calophya schini & Aleurodicus juleikae \\
\hline & & Aleurodicus coccolobae \\
\hline
\end{tabular}


Tabla 6. Tipo de daño y pieza bucal por insecto o ácaro y hospedero

\begin{tabular}{|c|c|c|c|}
\hline Hospedero & Insecto o ácaro & Tipo de piezas bucales & Tipo de daño \\
\hline \multirow{3}{*}{ Tecoma spp. } & Erosina hyberniata & Masticador & Hojas comidas, pudiendo afectar toda la copa. \\
\hline & Leptobyrsa decora & Picador- chupador & $\begin{array}{l}\text { Manchas de decoloración y Marchitez y caída prematura } \\
\text { de hojas. }\end{array}$ \\
\hline & Aleurodicus juleikae & Picador- chupador & $\begin{array}{l}\text { Succión de savia en hojas. } \\
\text { Reducción de la capacidad fotosintética. }\end{array}$ \\
\hline \multirow{3}{*}{ Eucalyptus spp. } & Aleurodicus juleikae & Picador- chupador & $\begin{array}{l}\text { Succión de savia en hojas. } \\
\text { Reducción de la capacidad fotosintética. }\end{array}$ \\
\hline & Glycaspis brimblecombei & Picador- chupador & $\begin{array}{l}\text { Succión de savia en hojas. } \\
\text { Caída prematura de hojas. } \\
\text { Reducción de la capacidad fotosintética. } \\
\text { Huecos en hojas por caída de lerps. }\end{array}$ \\
\hline & Sin identificación & Masticador & Comedura en hojas. \\
\hline \multirow{4}{*}{ Acacia retinoides } & Pseudolycaena nellyae & Masticador & Hojas comidas, pudiendo afectar toda la copa. \\
\hline & Marasmia tapezalis & Masticador & $\begin{array}{l}\text { Enrollador de hojas. } \\
\text { Comedura de parte superficial de la hoja. }\end{array}$ \\
\hline & Aleurodicus juleikae & Picador- chupador & $\begin{array}{l}\text { Succión de savia en hojas. } \\
\text { Reducción de la capacidad fotosintética. }\end{array}$ \\
\hline & Aspidiotus nerii & Picador- chupador & $\begin{array}{l}\text { Succión de savia en hojas. } \\
\text { Reducción de la capacidad fotosintética. }\end{array}$ \\
\hline \multirow{5}{*}{ Ficus benjamina } & Aleurodicus juleikae & Picador- chupador & $\begin{array}{l}\text { Succión de savia en hojas. } \\
\text { Reducción de la capacidad fotosintética. }\end{array}$ \\
\hline & Gynaikothrips uzeli & Picador- chupador & $\begin{array}{l}\text { Succión de savia en brotes. } \\
\text { Plegamiento de brotes. } \\
\text { Caída prematura de brotes. }\end{array}$ \\
\hline & Singhiella simplex & Picador- chupador & $\begin{array}{l}\text { Succión de savia en brotes. } \\
\text { Caída prematura de brotes. }\end{array}$ \\
\hline & Parasaissetia nigra & Picador- chupador & $\begin{array}{l}\text { Succión de savia en hojas. } \\
\text { Reducción de la capacidad fotosintética. }\end{array}$ \\
\hline & Ceroplastes cirripediformis & Picador- chupador & $\begin{array}{l}\text { Succión de savia en hojas. } \\
\text { Reducción de la capacidad fotosintética. }\end{array}$ \\
\hline \multirow{4}{*}{ Salix humboldtiana } & Schizotetranychus sp. & Raspador- chupador & $\begin{array}{l}\text { Raspado de la superficie de hoja. } \\
\text { Succión de savia en hojas. } \\
\text { Marchitez prematura. } \\
\text { Reducción de la capacidad fotosintética. }\end{array}$ \\
\hline & Sin identificación & Masticador & Comedura en hojas. \\
\hline & Aleurodicus coccolobae & Picador- chupador & $\begin{array}{l}\text { Succión de savia en hojas. } \\
\text { Reducción de la capacidad fotosintética. }\end{array}$ \\
\hline & Sin identificación & Masticador & Galería en hojas. \\
\hline \multirow{2}{*}{ Schinus molle } & Calophya schini & Picador- chupador & $\begin{array}{l}\text { Succión de savia en hojas. } \\
\text { Reducción de la capacidad fotosintética. }\end{array}$ \\
\hline & Aleurodicus juleikae & Picador- chupador & $\begin{array}{l}\text { Succión de savia en hojas. } \\
\text { Reducción de la capacidad fotosintética. }\end{array}$ \\
\hline Lagerstromia speciosa & Aleurodicus juleikae & Picador- chupador & $\begin{array}{l}\text { Succión de savia en hojas. } \\
\text { Reducción de la capacidad fotosintética. }\end{array}$ \\
\hline \multirow{3}{*}{ Tipuana tipu } & Platycorypha nigrivirga & Picador- chupador & $\begin{array}{l}\text { Succión de savia en ramas y peciolos y hojas tiernas. } \\
\text { Caída prematura de hojas. }\end{array}$ \\
\hline & Pinnaspis aspidistrae & Picador- chupador & $\begin{array}{l}\text { Succión de savia en hojas. } \\
\text { Reducción de la capacidad fotosintética. }\end{array}$ \\
\hline & Aleurodicus juleikae & Picador- chupador & $\begin{array}{l}\text { Succión de savia en hojas. } \\
\text { Reducción de la capacidad fotosintética*. }\end{array}$ \\
\hline Araucaria excelsa & Neophyllaphis araucariae & Picador- chupador & Succión de savia en hojas. \\
\hline \multirow{3}{*}{ Schinus terebinthifolius } & Aleurodicus juleikae & Picador- chupador & $\begin{array}{l}\text { Succión de savia en hojas. } \\
\text { Reducción de la capacidad fotosintética. }\end{array}$ \\
\hline & Pinnaspis aspidistrae & Picador- chupador & $\begin{array}{l}\text { Succión de savia en hojas. } \\
\text { Reducción de la capacidad fotosintética. }\end{array}$ \\
\hline & Parasaissetia nigra & Picador- chupador & $\begin{array}{l}\text { Succión de savia en hojas y ramas. } \\
\text { Reducción de la capacidad fotosintética. }\end{array}$ \\
\hline
\end{tabular}


Continuación Tabla 6.

\begin{tabular}{llll}
\hline Hospedero & Insecto o ácaro & Tipo de piezas bucales & Tipo de daño \\
\hline \multirow{2}{*}{ Spathodea campanulata } & Sin identificación & Picador- chupador & Succión de savia en hojas. \\
\cline { 2 - 3 } & Sin identificación & Masticador & Comedura en hojas. \\
\cline { 2 - 4 } Grevillea robusta & Aspidiotus nerii & Picador- chupador & $\begin{array}{l}\text { Succión de savia en hojas. } \\
\text { Reducción de la capacidad fotosintética. }\end{array}$ \\
\hline Cedrela odorata & Aleurodicus juleikae & Picador- chupador & $\begin{array}{l}\text { Succión de savia en hojas. } \\
\text { Reducción de la capacidad fotosintética. }\end{array}$ \\
\hline Swietenia macrophilla & Sin identificación & Masticador & Comedura en hojas. \\
\hline * Los insectos picadores- chupadores segregan una sustancia azucarada que incentiva el desarrollo de fumagina, hongo que al recubrir la superficie de las hojas \\
disminuye su capacidad fotosintética.
\end{tabular}

hojas, decoloración, daños en brotes, caída prematura de hojas, debilitamiento del árbol por succión de savia o disminución de la capacidad fotosintética, entre otros daños al follaje causados por insectos y por ácaros (Tabla 5). Es importante resaltar que el rango de número de insectos o ácaros que causan daño encontrado en los árboles fue de 1 a 5 (Tabla 4). La mayoría de hospederos presentaron de uno a tres insecto o ácaros causando daño; mientras Ficus benjamina, Acacia retinoides y Salix humboldtiana presentaron respectivamente 5,4 y 4. Este resultado sugiere que Ficus benjamina es la más vulnerable al daño de insectos y ácaros, resultado que debería ser tomado en cuenta en los programas de forestación urbana.

Las especies forestales que no presentaron daños ocasionados por insectos y ácaros durante el periodo de evaluación fueron las siguiente: Casuarina cunninghamiana, Cupressus macrocarpa, Fraxinus americana, Jacaranda mimosifolia, Melia azedarach, Enterolobium cyclocarpum, Koelreuteria paniculata, Delonix regia, Triplaris peruviana y Parkinsonia aculeata, las cuales representan un $42 \%$ de la muestra.

\section{Discusión}

De los insectos y ácaros identificados 14 insectos y 1 ácaro son por primera vez reportados en el país para sus hospederos forestales en el campus de la UNALM,(Tabla 2), mientras que Aleurodicus juleikae ha sido reportada por Valencia (2009) causando daños en el arbolado urbano limeño, además de ser la especie con mayor número de hospederos y Glycaspis brimblecombei había sido previamente reportada en la ciudad de Tacna (Burckhardt et al. 2008).

Tanto Platycorypha nigrivirga, Singhiella simplex y Neophyllaphis araucariae se reportan por primera vez en el Perú, siendo importante mencionar que Platycorypha nigrivirga es reconocida como una plaga importante en el arbolado urbano de Tipuana tipu en Argentina, Bolivia, España, Estados Unidos y Uruguay según Rung et al. (2009); Singhiella simplex es una plaga importante en Florida, Estados Unidos (Avery et al. 2011), cuyo origen, según Kondo (2012), se encuentra en la Región Oriental, Burma, China e India y ha sido introducida a los Estados Unidos (Florida) y Puerto Rico y además se le ha reportado en Jamaica, Brasil, Islas Canarias y Colombia y a Neophyllaphis araucariae se le reconoce como distribución geográfica Costa Rica, Australia, Hawaii, Mexico y Estados Unidos según Foottit et al. (2011).

Glycaspis brimbecombei se reportó por primera vez para Lima, Perú a través de la presente investigación y Burckhardt et al. (2008) lo reporta por primera vez en el Perú en Tacna.

De acuerdo al tipo de piezas bucales, los insectos y ácaros encontrados se dividieron en tres tipos: picadores-chupadores, masticadores y raspadorchupador (Tabla 5). El factor impulsor del ataque de estos insectos es la inadecuada práctica silvicultural de poda, principalmente. La poda que se realiza en el campus es una poda drástica que incentiva la aparición de brotes y hojas tiernas del hospedero que es de donde se alimentan estos insectos y ácaros. Además estas podas se realizan cada año, en cualquier época del año, y sin considerar la auto ecología del hospedero, cuyo resultado conduce a su debilitamiento. Otra práctica silvicultural que incentiva el ataque es el riego por inundación, porque pueden usarlo como medio, y debilita al hospedero al dejar al suelo sin espacios de aire y limitar su respiración.

De acuerdo a la Tabla 5 hay más especies de plagas monófagas que polífagas, por lo tanto se espera una mayor severidad de daño en los hospederos. Asimismo, el hecho de que haya un mayor número de especies de plagas picadores chupadores, ocasiona una mayor severidad de daño, porque al tener una metamorfosis paurometabola, o gradual, se alimentan de los árboles durante todo su ciclo biológico y debido 
a su corto ciclo de vida podemos encontrar varias generaciones coexistiendo en el mismo individuo. Sin embargo, hay que reconocer que los arboles estudiados (mayores de $10 \mathrm{~cm}$ ) son resistentes al ataque de estos diminutos insectos.

Los insectos y ácaros encontrados restan las funciones ornamentales y ambientales para lo cual fueron plantados los árboles.

De las 24 especies de árboles evaluados el $58 \%$ de ellas presentaron daños en el follaje. Estos resultados pueden ser explicados en función a que el follaje está más disponible para la mayoría de insectos y ácaros, así como, representa una gran cantidad de alimento. Además que la mayoría de las especies forestales que presentan daños en el follaje son siempreverdes o perennifolias, ya que al mantenerse con hojas durante gran parte del periodo de evaluación permiten el ataque de insectos o ácaros; mientras que las que no presentaron estos daños son especies caducifolias que renuevan periódicamente sus hojas, por lo que resulta ser una característica interesante a considerar cuando se establezcan planes de forestación urbana.

Los insectos y ácaros plagas claves en su mayoría causan daño a los hospederos con mayor número de individuos en el campus, esto es: Schinus molle, Eucalyptus spp., Ficus benjamina y Salix humboldtiana (Tabla 2), cuyo daño fue incrementado a partir del año 2010 a consecuencia de la forestación masiva en el campus de Tecoma spp. y Acacia retinoides. Por este motivo, de acuerdo a Manta (2013), es necesario diversificar el cultivo de árboles de un área antes de generar un plan de forestación y reforestación urbana, para evitar la generación, condiciones propicias para el establecimiento de plagas de ácaros e insectos.

Debido a que los hospederos se encuentran en abundancia y muchas veces agrupados en un mismo sitio (como arboledas o bermas) sobreponiendo las copas, cultivadas en lugares sombríos, o con inadecuada poda se contribuye al aumento del ataque de los insectos y ácaros plagas claves monófagas (Tabla 3), como el caso de: Erosina hyberniata, Calophya schini y Schizotetranychus sp.

Es importante mencionar a este nivel que es común encontrar hospederos con ataques de especies monófagas y polífagas al mismo tiempo durante la misma temporada. En la mayoría de esos casos los monófagos desplazan a los polífagos, como Erosina hyberniata (monófago) en Tecoma spp. que reduce el ataque de Aleurodicus juleikae (polífago) a plaga potencial o lo desplaza a otros hospederos.
En sentido contrario puede existir insectos polífagos que se comportan como plagas claves, y monófagas como potenciales en el mismo árbol, al mismo tiempo y durante la misma temporada, como es el caso de Aleurodicus juleikae y Gynaikothrips uzeli en Ficus benjamina. Esto se debe a que Aleurodicus juleikae se encontraba previamente establecida y con grandes poblaciones en brotes, hojas maduras y seniles aprovechando el lento recambio de hojas del hospedero, mientras que el ataque de Gynaikothrips uzeli recién iniciaba y se limitaba a brotes. Observaciones en el año 2015 posteriores a la evaluación indican que en ciertos árboles el ataque de Gynaikothrips uzeli desplazó al de Aleurodicus juleikae.

La presencia de cultivos ornamentales y agrícolas hospederas cultivadas en el campus de la UNALM es un factor importante para el desarrollo de ciertas plagas ocasionales y claves, caso Lantana cámara L. y Tecoma spp. con Leptobyrsa decora y Acacia retinoides y Pouteria lucuma (Ruiz \& Pav.) Kuntze con Pseudolycaena nellyae. En estos casos los insectos son plagas presentes y conocidas en los cultivos de Lantana camara y Pouteria lúcuma que encuentran en la especie forestal las condiciones favorables para su desarrollo.

En el caso de las plagas ocasionales, estas mayoritariamente se presentan cuando encuentran un factor favorable, como la temperatura, para su desarrollo. Como es el caso de Platycorypha nigrivirga en Tipuana tipu que se vio favorecido por el aumento de la temperatura durante el verano del año 2012, tal y como lo indica De Queiroz (2011).

En el verano del 2013 se encontró que Singhiella simplex en Ficus benjamina cambió su clasificación a plaga potencial, se debe posiblemente a que en el verano del 2012 recién iniciaba su ataque. Asimismo durante el primer y segundo trimestre del año 2016 se observó el ataque del ácaro Schizotetranychus sp., identificado por Huanca 2016, en Cedrela odorata y el ataque de Paracoccus marginatus, identificado por Díaz 2016, en la especie de Tecoma spp.

\section{Conclusiones}

- El daño registrado en el arbolado del campus de la UNALM es en el follaje de especies mayoritariamente siempreverdes y es causado principalmente por insectos picadores chupadores en una proporción similar entre insectos monófagos y polífagos. 
- Ficus benjamina es la especie forestal que presenta mayor número de ataques (cinco insectos picadores chupadores).

- $\quad$ Aleurodicus juleikae es la especie insectil con el mayor número de hospederos (ocho).

- Los insectos monófagos estudiados tienden a comportarse como plagas claves y posiblemente disminuyen el ataque de insectos polífagos.

- De acuerdo a los valores de incidencia y severidad y su duración las plagas claves son: Erosina hyberniata, Aleurodicus juleikae en Ficus benjamina, Eucalyptus spp., Lagerstromia speciosa, Calophya schini, Pseudolycaena nellyae y Schizotetranychus sp. Las plagas potenciales son: Aleurodicusjuleikae en Schinus terebinthifolius y Acacia retinoides, Aspidiotus nerii en Grevillea robusta, Marasmia tapezalis, agallas en las hojas en Spathodea campanulata, Gynaikothrips uzeli, comeduras en hojas en Cedrela odorata y Salix humboldtiana. Pinnaspis aspidistrae en Schinus terebinthifolius. Las plagas esporádicas u ocasionales son: Neophyllaphis araucariae, Platycorypha nigrivirga, Glycaspis brimblecombei y Leptobyrsa decora.

\section{Agradecimientos}

Al Dr. Axel Hausmann, del Museo de Munich Alemania por la identificación de Erosina hyberniata. Al Dr. Gregory Evans, de Animal and Plant Health Inspection Service (APHIS) Estados Unidos, por la identificación de Singhiella simplex. Al Dr. Laurence Mound, de Commonwealth Scientific and Industrial Research Organization (CSIRO), Australia, por la identificación de Gynaikothrips uzeli. A la Ing. Lourdes Peralta, Servicio Agrícola y Ganadero de Chile, por la identificación de Schizotetranychus sp. y al Blgo. Walter Diaz por su colaboración en cuanto a la identificación.

Asimismo, también quisiéramos agradecer al Ing. Giancarlo Orellana por el apoyo en las colectas y evaluaciones y a la Dra. María Isabel Manta por los comentarios y sugerencias.

\section{Literatura citada}

Agrios, G.

2002. Fitopatología. 7ma ed. Limusa. Distrito Federal de México, MX. 839 p.

Alata, J.

1973. Lista de insectos y otros animales dañinos a la agricultura en el Perú. Ministerio de Agricultura. PERU. 176p.

Avery, P; Mannion, C; Powell, C; Mckenzie, C \& Osborne, L. 2011. Natural Enemies Managing the Invasion of the Fig Whitefly, Singhiella simplex (Hemiptera: Aleyrodidae), Infesting a Ficus benjamina Hedge. Florida Entomologist, 94 (3): 696-699.

Burckhardt, D; Lozada, P. \& Diaz, W.

2008. First record of the red gum lerp psyllid Glycaspis brimblecombei (Hemiptera: Psylloidea) from Peru. Bulletin de la Société Entomologique Suisse, 81: 83-85.

De Queiroz, D.

2011. Psílideos no Brasil 6: Psílídeo da tipuana. Comunicado técnico, 284 (1980-1982): 1-3 p

Delgado, C \& Couturier, G.

2004. Manejo de insectos plagas en la Amazonía: Su aplicación en Camu camu; IIAP-IRD. Lima, PERU. 147p.

Dourojeanni, R.

1963. Introducción al estudio de los insectos que afectan la explotación Forestal de la selva peruana. Revista Peruana de Entomología Agrícola, 6 (1): 27-38.

Foottit, R; Maw, H; Pike, K \& Messing, R.

2011. The aphids (Hemiptera: Aphididae and Adelgidae) of Hawaii: Annotated list and key to species of an adventive fauna. Pacific Science, 66 (1): 1-85.
Kondo, T.

2012. Singhiella simplex (Singh) (Hemiptera: Aleyrodidae), a new aleyrodid invasive species for Colombia. Boletín del Museo de Entomología de la Universidad del Valle, 13 (2): 31-33.

Manta, MI.

2013. Estimación de daños provocados por insectos en las plantaciones forestales, en el contexto del cambio climático. LV Convención de Entomología. Sociedad Entomológica del Perú. Universidad Nacional Agraria La Molina, Perú. Del 4 al 7 de noviembre del 2013.

Pérez, T.

1999. La especialización en los insectos fitófagos: una regla más que una excepción. Evolución y Filogenia de Artropodos, 26: 759-776.

Rung, A; Arakelian, G; Gill, R y Nisson, N.

2009. Platycorypha nigrivirga Burckhardt (Hemiptera: Sternorrhyncha: Psylloidea), tipu psyllid, new to North America. Insecta Mundi, 2009 (0097): 1-5.

SENASA.

2006. Manual Procedimiento de Muestreo y Toma y Envío de Muestras de Artropodos de Importancia Forestal. Servicio De Sanidad Agraria. Lima, Perú. 15p.

SENASA.

2008. Hongos e Insectos Forestales en el Perú: Identificación e Inventario de Insectos, Hongos y Cromistas de Importancia Forestal en las Regiones de Huánuco, Junín, Lima, Loreto, Madre de Dios, San Martín y Ucayali. Servicio De Sanidad Agraria. Lima, Perú. 41 p. 